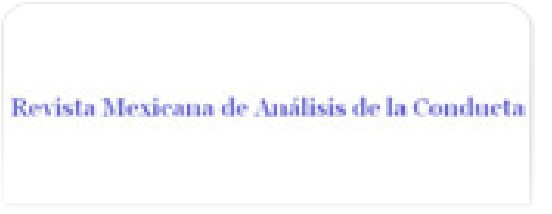

Revista Mexicana de Análisis de la Conducta ISSN: 0185-4534

editora@rmac-mx.org

Sociedad Mexicana de Análisis de la Conducta México

Torres, Alvaro; López, Florente

Discriminación condicional de la propia conducta, verbalización de contingencias y relaciones condicionales emergentes

Revista Mexicana de Análisis de la Conducta, vol. 30, núm. 2, diciembre, 2004, pp. 139-162 Sociedad Mexicana de Análisis de la Conducta

Guadalajara, México

Disponible en: http://www.redalyc.org/articulo.oa?id=59330202

Cómo citar el artículo

- Número completo

- Más información del artículo

Página de la revista en redalyc.org

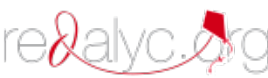

Sistema de Información Científica

Red de Revistas Científicas de América Latina, el Caribe, España y Portugal Proyecto académico sin fines de lucro, desarrollado bajo la iniciativa de acceso abierto 


\title{
DISCRIMINACIÓN CONDICIONAL DE LA PROPIA CONDUCTA, VERBALIZACIÓN DE CONTINGENCIAS Y RELACIONES CONDICIONALES EMERGENTES
}

\author{
CONDITIONAL DISCRIMINATION OF ONE'S OWN \\ BEHAVIOR, CONTINGENCY VERBALIZATION, AND EMERGENT \\ CONDITIONAL RELATIONSHIPS
}

ALVARO TORRES Y FLORENTE LÓPEZ

UNIVERSIDAD NACIONAL AUTÓNOMA DE MÉXICO

\begin{abstract}
RESUMEN
Se analizaron los efectos del preentrenamiento en identificación y verbalización de relaciones contingentes y del nombramiento concurrente de contingencias, sobre la adquisición de una discriminación condicional de la propia conducta y sobre la formación de relaciones condicionales emergentes indicativas de equivalencia. Para ello, 40 participantes universitarios se asignaron a cuatro grupos: sin preentrenamiento y sin verbalización; con preentrenamiento y sin verbalización; sin preentrenamiento y con verbalización; y, con preentrenamiento y con verbalización simultánea. Se encontraron diferencias en la velocidad de adquisición de la discriminación mencionada, y en el número de pruebas de relaciones condicionales emergentes aprobadas. Estas diferencias sugieren que la conducta verbal autodescriptiva facilita la adquisición de una discriminación condicional de la propia conducta y la formación de relaciones condicionales emergentes indicativas de equivalencia.
\end{abstract}

1. Favor de enviar toda correspondencia relacionada con el artículo al primer autor al correo electrónico: alvarot@servidor.unam.mx. Esta investigación se realizó como parte de los estudios de Doctorado en Psicología del primer autor y recibió apoyo de la Beca \# 158986 del CONACYT. Los autores agradecen al Dr. François Tonneau y a otros dos revisores anónimos sus comentarios al manuscrito de este artículo. 
Palabras Clave: equivalencia de estímulos, nombramiento de contingencias, discriminación condicional de la propia conducta, relaciones condicionales emergentes, adultos jóvenes.

\begin{abstract}
We analyzed the effects of pre-training in the identification and verbalization of contingent relationships and the concurrent naming of contingencies, on the acquisition of a conditional discrimination of the subject's own behavior and on the formation of emergent conditional relationships indicative of equivalence. With this purpose, 40 college students were randomly assigned to one of four groups: Control, no training-no verbalization, training-no verbalization, no training-verbalization, and training-simultaneous verbalization. Differences were found in the number of trials required for the attainment of discrimination and in the correct number of probes of emergent conditional relations. These differences suggest that the self-descriptive verbal behavior promotes the acquisition of the conditional discrimination of the subject's own behavior and of the formation of emergent conditional relations indicative of equivalence.

Key words: Stimulus equivalence, contingency naming, conditional discrimination of the subject's own behavior, emergent conditional relationships, young adult humans.
\end{abstract}

Discriminación condicional, equivalencia de estímulos y nombramiento son tres áreas de investigación del Análisis Experimental de la Conducta que han confluido en el análisis y explicación del papel que juega la conducta verbal, característica de los humanos, en la determinación de la conducta no-verbal y de las llamadas conductas complejas o superiores.

En la discriminación condicional el significado de los estímulos discriminativos cambia en función del contexto en el que aparecen; la respuesta correcta debe basarse en las propiedades de dos o más estímulos. El paradigma de igualación de la muestra constituye una tarea representativa de la discriminación condicional (Cumming y Berryman, 1965). En igualación de la muestra, cuál de dos o más estímulos de comparación será positivo (es decir, la respuesta a cuál de esos estímulos será reforzada) depende de las propiedades del estímulo muestra.

La igualación de la muestra puede ser de identidad, cuando los estímulos de muestra y de comparación son iguales; o puede ser arbitraria o simbólica, cuando los estímulos de muestra y de comparación tienen propiedades físicas completamente diferentes pero mantienen una relación arbitraria, socialmente convenida. 
Pero, como han señalado Sidman y colaboradores (1982) y Sidman y Tailby (1982), la simple observación de las ejecuciones de discriminación condicional de un sujeto, no permite decir si involucran o no involucran respuestas de igualación de la muestra verdaderas. Para ello se debe demostrar que los estímulos, involucrados en relaciones condicionales del tipo "Si...entonces", también satisfacen las propiedades de una relación de equivalencia: es decir, que poseen las propiedades de reflexividad, simetría y transitividad.

La reflexividad se refiere a que un estímulo mantiene una relación condicional consigo mismo (aRa); la simetría requiere que haya bidireccionalidad entre dos estímulos diferentes ( $\mathrm{Si}$ aRb, entonces $\mathrm{bRa}$ ); la transitividad requiere que la relación se mantenga entre tres estímulos, para probar esta propiedad se necesita que el sujeto adquiera una segunda discriminación condicional $b R c$ además de $a R b$, de tal forma que demuestre que si $a R b$ y $\mathrm{bRc}$, entonces las relaciones condicionales aRc y cRa deben emerger en el repertorio del sujeto sin haberse entrenado directamente.

Cuando se demuestra que las relaciones entre los estímulos poseen esas tres propiedades, se dice que las relaciones condicionales emergentes son indicativas de equivalencia de estímulos y que los estímulos forman una clase equivalente cuyos integrantes significan lo mismo para el sujeto, a pesar de que sus propiedades sean completamente distintas.

Una explicación de por qué emergen relaciones condicionales no entrenadas directamente vía reforzamiento, propone que esto se debe a que los sujetos aplican nombres comunes a los estímulos de muestra y de comparación positivos (Horne y Lowe, 1996).

La definición conductual del término nombramiento según Catania (1988), se refiere a: "una clase conductual de orden superior que involucra clases estímulo arbitrarias (cosas o eventos con nombres particulares) y sus correspondientes topografías verbales arbitrarias (las palabras que sirven como sus nombres) en una relacional bidireccional. Los prerrequisitos del nombramiento incluyen al menos tres componentes: (i) la conducta del escucha, al mirar cosas y señalarlas con base en lo que se ha dicho; (ii) la conducta ecoica, al repetir los nombres cuando son expresados; y (iii) el tacto, al decir los nombres dados a los objetos" (p. 398).

Las relaciones entre discriminación condicional, relaciones indicativas de equivalencia y nombramiento se han estudiado desde hace tiempo. En los trabajos pioneros de Sidman y colaboradores (Constantine y Sidman, 1975; Sidman, 1971; Sidman y Cresson, 1973; Sidman, Cresson y Willson-Morris, 1974), sobre la emergencia de relaciones condicionales no entrenadas en el repertorio de sujetos humanos, se observó que éstos emiten conducta verbal de modo espontáneo y concurrente con sus ejecuciones: los sujetos suelen nombrar los eventos estímulo de las tareas experimentales.

Este hecho, posiblemente, ha llevado a algunos autores (Dugdale y 
Lowe, 1990; Horne y Lowe, 1996; Lowe, 1979, 1983) a proponer que el nombramiento es un requisito necesario y suficiente para la ocurrencia de esas relaciones condicionales emergentes. Esta propuesta ha sido controvertida.

Unos autores han demostrado que nuevas relaciones condicionales pueden emerger, aún en ausencia de nombramiento o de entrenamiento en relaciones condicionales auditivo-visuales que podrían promover el aprendizaje de nombres para los estímulos (Lazar, Davis-Lang y Sánchez, 1984; Sidman, 1992; Sidman y Tailby, 1982; Sidman, Willson-Morris y Kirk, 1986).

Otros autores como Eikeseth y Smith (1992) han demostrado que sujetos que no habían logrado formar relaciones de igualación de la muestra indicativas de equivalencia, lo pudieron hacer cuando se les enseñó a asignar el mismo nombre a todos los miembros potenciales de cada clase equivalente; Rehfeldt y Dixon (2000) han concluido que el hecho de describir abiertamente la propia ejecución incrementa la probabilidad de que se formen relaciones estímulo; Sato (2001), mostró que el nombramiento de los estímulos de muestra, durante el período de demora en pruebas de igualación de la muestra demorada, puede facilitar el establecimiento de equivalencia de estímulos.

Este conjunto de investigaciones no ha demostrado que el nombramiento sea necesario para la formación de equivalencias; sin embargo, sus datos indican un papel facilitador del nombramiento en la emergencia de nuevas relaciones condicionales indicativas de equivalencia.

Por otra parte, Ribes, Torres y Ramírez (1996) han mostrado efectos de distintos modos de descripción verbal de la ejecución y de las relaciones estímulo sobre la adquisición, la transferencia y el mantenimiento de una discriminación condicional. Sus datos indican que los sujetos que leyeron, copiaron o reconocieron las descripciones tuvieron altos porcentajes de ejecución en las pruebas de transferencia y en la posprueba.

Ribes y Castillo (1998), y Ribes, Moreno y Martínez (1998), emplearon un procedimiento de 'entrenamiento observacional', en el que los estímulos de igualación (figuras arbitrarias de colores) se presentaron junto con un texto descriptivo (el nombre de las figuras debajo de ellas), que los sujetos tenían que leer en voz alta en cada ensayo. Los autores señalan que los "efectos del entrenamiento observacional parecen mínimos", aunque podrían haber tenido cierto papel al complementar la influencia de las descripciones verbales construidas por los sujetos. Sus resultados indican un efecto facilitador de las respuestas de igualación verbal (consistentes en la elección de textos descriptivos de los estímulos y sus relaciones) sobre la adquisición y transferencia de discriminaciones condicionales.

Además de si el nombramiento, la verbalización o el tacteo a los estímulos tienen influencia sobre las discriminaciones condicionales emergentes, puede haber muchos otros factores. Uno de ellos, de interés particular para 
este estudio, puede ser la complejidad y arbitrariedad de los estímulos y relaciones empleados en las tareas experimentales de igualación de la muestra.

Algunos de los estudios mencionados anteriormente usaron palabras y dibujos de objetos comunes como estímulos para el establecimiento y la prueba de relaciones condicionales; sin embargo, la mayoría de los estudios sobre emergencia de relaciones condicionales han utilizado estímulos y relaciones arbitrarios (p.e., relaciones entre figuras geométricas de colores distintos, entre formas negras arbitrarias, entre palabras y letras griegas mayúsculas y minúsculas, entre palabras en idiomas desconocidos por los sujetos, y relaciones entre estímulos de segundo orden).

Como han señalado Randell y Remington (1999), es posible que ese tipo de relaciones arbitrarias sea incongruente con las estrategias que usan los sujetos para relacionar y describir los estímulos de muestra y de comparación; y, que esa incongruencia dificulte el surgimiento de nuevas relaciones condicionales.

Por ello, en este reporte se propone una tarea de discriminación condicional de la propia conducta. En esta discriminación, los sujetos construyen y determinan con su ejecución (bajo contingencias de intervalo y de razón), las propiedades visuales del estímulo muestra (una gráfica acumulativa de su ejecución); aunque en realidad lo que constituye el estímulo muestra, es su patrón de conducta de presionar una tecla de computadora (con distintas propiedades específicas de contexto, de frecuencia, de duración y de tiempo entre respuestas), que el sujeto puede experimentar y probar. La gráfica constituye un producto más o menos permanente de su propia conducta, pero resalta visualmente ciertas propiedades discriminativas del patrón de ejecución.

Este tipo de preparación experimental permite que el sujeto utilice su conocimiento de las relaciones entre su conducta y el ambiente experimental que le son familiares, dado que él mismo las establece durante el proceso de adquisición de una discriminación condicional. Este conocimiento, posiblemente en forma de auto-descripciones verbales, puede coadyuvar a la emergencia de relaciones condicionales no entrenadas directamente.

La tarea de discriminación de la propia conducta permite, además, que el experimentador observe los patrones objetivos de conducta no-verbal y que los pueda relacionar con diversas manipulaciones experimentales; por ejemplo, con instrucciones o auto-descripciones acerca de los estímulos y las contingencias que los sujetos pueden recibir o construir en su interacción con los procedimientos experimentales. Esta preparación integra la ejecución bajo programa y la discriminación condicional en el mismo dispositivo analítico.

Así pues, los objetivos del presente experimento fueron: proponer formalmente dicha tarea de discriminación condicional de la propia conducta; así como evaluar los efectos del preentrenamiento en identificación y verbaliza- 
ción de relaciones contingentes y de la verbalización simultánea de contingencias, sobre la adquisición de una discriminación condicional de la propia conducta y sobre la emergencia de relaciones condicionales indicativas de equivalencia.

\section{MÉTODO}

\section{Participantes}

Participaron voluntariamente cuarenta estudiantes de Psicología, 34 mujeres y 6 hombres, con una edad de entre 18 y 24 años de edad, (promedio 19.5 años), sin experiencia en las tareas dispuestas en este experimento. Obtuvieron créditos parciales en cursos universitarios a cambio de su participación.

\section{Materiales y situación experimental}

Se empleó una computadora personal Digital "Venturis" $466^{\circledR}$, con monitor de color y teclado. Las instrucciones y las tareas experimentales se presentaron en la pantalla del monitor. Las respuestas se registraron automáticamente a través del sistema de cómputo. Los programas de presentación de tareas y de registro de datos se elaboraron con Turbo Pascal $6.0^{\odot}$. Se utilizaron hojas de papel y lápices. Las sesiones experimentales fueron individuales, tuvieron una duración promedio de 25 minutos y se realizaron diariamente en un cubículo de $5 \times 3$ metros, con un escritorio y una computadora, ante la presencia del experimentador.

\section{Diseño}

Se utilizó un diseño factorial $2 \times 2$ (Kerlinger, 1975; Keppel, Saufley, Jr. y Tokunaga, 1992). Un factor, preentrenamiento en identificación y verbalización de contingencias, tuvo dos niveles: con y sin preentrenamiento. El otro factor, verbalización simultánea de las contingencias de la adquisición de una discriminación condicional de la propia conducta, también estuvo compuesto por dos niveles: con y sin verbalización. Los sujetos se asignaron al azar a cuatro grupos de 10 sujetos cada uno.

\section{Procedimiento}

El experimento consistió de tres condiciones a las que se sometió a los sujetos: preentrenamiento en identificación y verbalización de contingencias; adquisición de una discriminación condicional de la propia conducta; y, prue- 
ba de relaciones condicionales emergentes. La primera condición se aplicó sólo a dos grupos experimentales y las dos últimas condiciones se aplicaron a todos los grupos experimentales (véase la Tabla 1).

Tabla 1. Condiciones experimentales a que fueron sometidos los sujetos de los grupos experimentales.

\begin{tabular}{|c|c|c|c|c|c|}
\hline & \multicolumn{5}{|c|}{ Condiciones experimentales } \\
\hline \multirow[t]{2}{*}{ Grupos } & \multicolumn{2}{|c|}{$\begin{array}{l}\text { 1. Preentrenamiento en } \\
\text { identificación y verbalización de } \\
\text { eventos }\end{array}$} & \multicolumn{2}{|c|}{$\begin{array}{l}\text { 2. Adquisición de una } \\
\text { discriminación condicional de la } \\
\text { propia conducta }\end{array}$} & \multirow{2}{*}{$\begin{array}{l}\text { 3. Pruebas } \\
\text { de relaciones } \\
\text { condicionales } \\
\text { emergentes } \\
\text { indicativas de } \\
\text { equivalencia }\end{array}$} \\
\hline & $\begin{array}{c}\text { Con pre- } \\
\text { entrenamiento }\end{array}$ & $\begin{array}{c}\text { Sin pre- } \\
\text { entrenamiento }\end{array}$ & $\begin{array}{c}\text { Con } \\
\text { verbalización } \\
\text { de eventos } \\
\end{array}$ & $\begin{array}{c}\text { Sin } \\
\text { verbalización } \\
\text { de eventos } \\
\end{array}$ & \\
\hline SE-SV & & $\checkmark$ & & $\checkmark$ & $\checkmark$ \\
\hline E-SV & $\checkmark$ & & & $\checkmark$ & $\checkmark$ \\
\hline SE-V & & $\checkmark$ & $\checkmark$ & & $\checkmark$ \\
\hline E-V & $\checkmark$ & & $\checkmark$ & & $\checkmark$ \\
\hline \multicolumn{6}{|c|}{ 飞a = condición aplicada } \\
\hline
\end{tabular}

Preentrenamiento en identificación y verbalización de relaciones contingentes. En esta primera condición experimental, se entrenó a los participantes en una tarea de identificación y verbalización de las relaciones contingentes entre dos secuencias de colores y dos tonos de diferente duración.

El preentrenamiento se realizó en una sesión de 25 minutos de duración, de la siguiente manera (véase la Tabla 2). Inicialmente, se sentó a los sujetos en un escritorio frente a una computadora y en el monitor se les mostraron las instrucciones generales de la tarea de preentrenamiento, mismas que el experimentador leyó en voz alta a la vez que los sujetos las leían. La presentación de las instrucciones y de los ensayos subsecuentes terminaron hasta que el sujeto presionó la «barra espaciadora» 
Tabla 2. Instrucciones generales y específicas, estímulos presentados y actividades realizadas por los participantes de los grupos con preentrenamiento en identificación y verbalización de relaciones contingentes.

\begin{tabular}{|c|c|c|c|c|c|c|}
\hline $\begin{array}{l}\text { En pri } \\
\text { En se } \\
\text { En ter } \\
\text { Y, por } \\
\text { ¿Tien }\end{array}$ & $\begin{array}{l}\text { ner lugar, lo que } \\
\text { undo lugar, deb } \\
\text { er lugar, deber } \\
\text { simo, respond } \\
\text { s alguna duda? }\end{array}$ & $\begin{array}{l}\text { Ins } \\
\text { enes que hac } \\
\text { ás describir e } \\
\text { decir en voz } \\
\text { a la pregunta } \\
\text { tila al experim }\end{array}$ & $\begin{array}{l}\text { rucciones gener } \\
\text { r es poner mucha } \\
\text { voz alta todo lo } \\
\text { ta qué está relaci } \\
\text { que se mostrará e } \\
\text { tador ahora. }\end{array}$ & $\begin{array}{l}\text { les: } \\
\text { atención a todo } \\
\text { ue veas y escuc } \\
\text { nado con qué. } \\
\text { la pantalla. }\end{array}$ & $\begin{array}{l}\text { lo que veas y } \\
\text { hes. }\end{array}$ & escuches. \\
\hline Bloque e & & & Combinaciones & e estímulos & & \\
\hline $\begin{array}{c}\text { instrucción } \\
\text { específica }\end{array}$ & $\begin{array}{l}\text { Cantidad de } \\
\text { ensayos }\end{array}$ & $\begin{array}{c}\text { Figura } \\
\text { geométrica }\end{array}$ & $\begin{array}{c}\text { Secuencia de } \\
\text { colores * }\end{array}$ & $\begin{array}{l}\text { Posición de } \\
\text { presentación }\end{array}$ & $\begin{array}{l}\text { Duración } \\
\text { del tono }\end{array}$ & $\begin{array}{l}\text { Actividad del } \\
\text { participante }\end{array}$ \\
\hline & & Cuadrados & Az-V-R-Am & Horizontal & Largo & \\
\hline & & Cuadrados & Am-R-V-Az & Horizontal & Corto & \\
\hline Bloque \# 1 & & Cuadrados & $A z-V-R-A m$ & Vertical & Largo & \\
\hline "Atiende a todo & 8 & Cuadrados & $A m-R-V-A z$ & Vertical & Corto & Ver y escuchar \\
\hline lo que veas y & 8 & Círculos & Az-V-R-Am & Horizontal & Largo & todos los eventos \\
\hline escuches" & & Círculos & $A m-R-V-A z$ & Horizontal & Corto & \\
\hline & & Círculos & Az-V-R-Am & Vertical & Largo & \\
\hline & & Círculos & $A m-R-V-A z$ & Vertical & Corto & \\
\hline $\begin{array}{c}\text { Bloque \# } 2 \\
\text { "Di en voz alta } \\
\text { todo lo que } \\
\text { pasa" }\end{array}$ & 8 & & Igual que en el bl & que anterior & & $\begin{array}{l}\text { Describir en voz } \\
\text { alta lo que veian y } \\
\text { escuchaban }\end{array}$ \\
\hline $\begin{array}{l}\text { Bloque \# } 3 \\
\text { "Di en voz } \\
\text { alta qué está } \\
\text { relacionado } \\
\text { con qué" }\end{array}$ & 8 & & Igual que en el bl & que anterior & & $\begin{array}{l}\text { Decir en voz alta } \\
\text { qué se relaciona } \\
\text { con qué }\end{array}$ \\
\hline $\begin{array}{l}\text { Identificación de } \\
\text { Ahora lo que tie } \\
\text { estaba ocurrien } \\
\text { a) cuadrados co } \\
\text { b) Secuencia d } \\
\text { verde y azul cor } \\
\text { c) Posición hori } \\
\text { Selecciona una }\end{array}$ & $\begin{array}{l}\text { Relaciones: } \\
\text { hes que hacer } \\
\text { o. } \\
\text { i sonido largo y } \\
\text { colores azul, } \\
\text { tono corto. } \\
\text { ontal con tono I } \\
\text { le las opciones }\end{array}$ & $\begin{array}{l}\text { elegir, de las } \\
\text { rculos con sor } \\
\text { de, rojo y am } \\
\text { go y posición } \\
\text { teriores, y pre }\end{array}$ & $\begin{array}{l}\text { opciones siguien } \\
\text { ido corto. } \\
\text { rillo con tono larg } \\
\text { ertical con tono co } \\
\text { siona la letra corr }\end{array}$ & $\begin{array}{l}\text { es, la relación } \\
\text { y secuencia } \\
\text { rto. } \\
\text { spondiente. }\end{array}$ & $\begin{array}{l}\text { ue supones } \\
\text { marillo, rojo, }\end{array}$ & $\begin{array}{l}\text { Leer la pregunta } \\
\text { y elegir una de } \\
\text { las opciones } \\
\text { presionando } \\
\text { la tecla } \\
\text { correspondiente }\end{array}$ \\
\hline & & ${ }^{2} A z=A z u l, V$ & $=$ Verde, $\mathrm{R}=$ Rojo & Am = Amarillo & & \\
\hline
\end{tabular}

Luego se les dieron tres bloques de ocho ensayos. En el primer bloque se les mostraron, de manera secuencial ( 0.5 segundos entre cada figura), cuatro cuadrados o cuatro círculos de colores azul, rojo, verde y amarillo, o estos colores en orden inverso; seguidos 0.2 segundos después por un tono corto $(500 \mathrm{~Hz}$. y $0.5 \mathrm{seg}$. de duración) o largo $(500 \mathrm{~Hz}$. y $1.5 \mathrm{seg}$. de duración) que sonaba en la bocina de la computadora. En cada ensayo de este bloque, apareció escrita en la parte superior de la pantalla la instrucción "Pon atención a todo lo que veas y escuches". En el segundo bloque, se les mostraron los mismos estímulos que en el bloque anterior, con la instrucción: "Ahora di en voz alta todo lo que veas y escuches". En el tercer bloque, con los mismos estímulos, la instrucción fue: "Di qué está relacionado con qué". En este bloque los sujetos, además de decir lo que veían y escuchaban en la computadora, también indicaron lo que ellos creían que estaba relacionado. 
Por último en esta condición de preentrenamiento en identificación y verbalización de relaciones contingentes, se presentó a los sujetos una pregunta con tres opciones de respuesta que describían únicamente tres de las relaciones posibles. Las dos relaciones contingentes correctas fueron: la relación de la secuencia de colores azul, rojo, verde y amarillo con el tono largo; y la relación de la secuencia de colores amarillo, verde, rojo y azul con el tono corto. Cuando los sujetos no eligieron la opción correcta, al día siguiente se les dio una nueva sesión completa hasta que seleccionaron la opción correcta. Los sujetos no recibieron reforzamiento ni retroalimentación en cada ensayo, sólo se les dio información hasta que lograron el criterio (elegir la opción 'b'); se pidió su asistencia al día siguiente para iniciar la segunda condición.

Adquisición de una discriminación condicional de la propia conducta. En esta segunda condición experimental se dispuso la adquisición de una discriminación condicional de la propia conducta, en un formato semejante al procedimiento convencional de igualación de la muestra, usual en los estudios de equivalencia (véase la Figura 1).

Cada sesión tuvo una duración aproximada de 30 minutos, en los cuales hubo hasta 30 ensayos, la mitad bajo una contingencia de intervalo fijo 2 seg. y la otra mitad con una contingencia de razón fija 5; la secuencia de los ensayos fue la misma para todos los participantes. Se realizaron tantas sesiones como fueron requeridas por los participantes para alcanzar el criterio. Para la segunda y posteriores sesiones, cuando fueron necesarias, se usó una nueva secuencia de ensayos también determinada aleatoriamente, pero cuidando que siempre fuera distinta a la secuencia de la sesión previa.

Las sesiones de esta segunda condición experimental comenzaron nuevamente con la presentación de las instrucciones generales, las cuales se mostraron por escrito en el monitor y fueron las siguientes:

"En este estudio se pretende analizar algunos de los factores que se relacionan con la conducta humana en situaciones de laboratorio. La actividad que vas a realizar en este estudio se divide en dos componentes: uno de EJECUCIÓN y otro de REPORTE. A lo largo de una sesión, estos dos componentes se repetirán 30 veces.

En el componente de EJECUCIÓN, para ganar puntos, tendrás que oprimir la tecla 'Flecha Abajo'. Podrás oprimir la tecla de la manera que quieras: rápido; despacio; esperar y luego apretar rápido; apretar rápido, esperar y luego volver a apretar; etc.

En el componente de ELECCIÓN, para ganar puntos, tendrás que oprimir la tecla 'Flecha Izquierda', o bien la tecla 'Flecha Derecha', según consideres pertinente".

Luego de la lectura de estas instrucciones, los participantes dieron inicio a los ensayos experimentales. Cada ensayo tuvo dos componentes: uno de ejecución y uno de elección. En el componente de ejecución, los participan- 


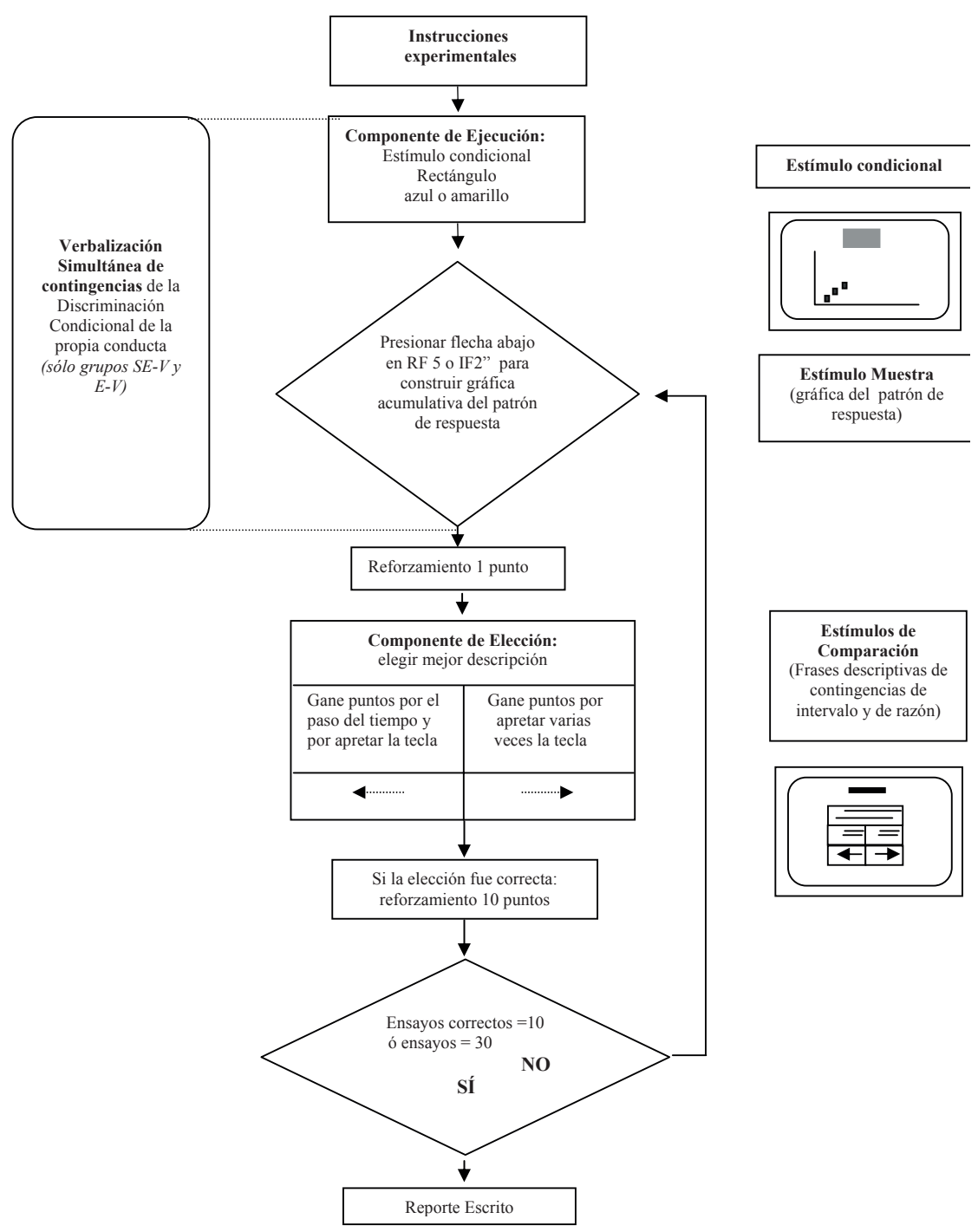

Figura 1. Estructura de una discriminación condicional de la propia conducta. En el lado derecho de la figura, se muestran dos ejemplos de las pantallas que se les presentaron a los sujetos. 
tes apretaron la tecla "flecha abajo" de una computadora ante la presencia de un estímulo condicional (un rectángulo de color azul o un rectángulo de color amarillo), que aparecía en medio de la parte superior del monitor cromático.

Los estímulos condicionales estuvieron asociados, respectivamente, a una contingencia de razón (RF5) o a una contingencia de tiempo (IF 2"). Junto con el estímulo condicional aparecían, en la parte inferior de la pantalla, los ejes de una gráfica. Cada pulsación de la tecla "flecha abajo" producía un punto en una gráfica acumulativa que el sujeto veía en el monitor. En otras palabras, el participante construía con sus respuestas una gráfica acumulativa de su propia conducta, que se constituía en el estímulo discriminativo de la relación condicional. Al cumplir con el requisito programado de razón o de intervalo, el sujeto recibía un punto y pasaba al componente de elección. En el componente de elección se presentaron en la pantalla la instrucción "Elige la frase que describe mejor lo que acabas de hacer para ganar puntos. Presiona flecha derecha o izquierda" y dos frases que constituían los estímulos de comparación:

"Gané puntos por el paso del tiempo y por apretar la tecla y Gané puntos por apretar varias veces la tecla".

Estas opciones se presentaron de modo aleatorio en las posiciones izquierda y derecha de la pantalla. Debajo de cada frase se mostró el símbolo de una flecha dirigida hacia la izquierda o hacia la derecha, para señalar a los participantes que deberían presionar la tecla "flecha izquierda" o la tecla "flecha derecha", para elegir la opción que a su juicio describiera mejor la contingencia que acababa de estar activa en el componente de ejecución. La función de esas frases, como estímulo de comparación positivo o negativo, dependió de cual hubiese sido el estímulo condicional y el patrón de respuesta que el propio sujeto hubiese construido en la gráfica acumulativa. Las elecciones correctas fueron reforzadas con diez puntos y la aparición de un letrero que decía "Elegiste la flecha (izquierda o derecha). Ganaste 10 puntos. Puntos acumulados $=x x^{\prime \prime}$. Las elecciones incorrectas fueron castigadas con la pérdida de diez puntos y la aparición de un letrero que decía "Elegiste la flecha (izquierda o derecha). Dejaste de Ganar 10 puntos. Puntos acumulados $=x x$ ". La elecciones incorrectas no restaban puntos a los acumulados por el sujeto, simplemente perdían la oportunidad de ganar puntos.

La tarea de discriminación de la propia conducta concluyó toda vez que los sujetos lograron un criterio de diez elecciones correctas continuas; por lo menos cinco en los ensayos de razón y cinco en los ensayos de tiempo. Al término de cada sesión los participantes realizaron un reporte escrito en respuesta a la instrucción:

"En la hoja que se te entregó, escribe por favor, QUÉ HICISTE y en qué te fijaste para ganar puntos y para lograr reportes correctos."

Finalmente, se dio información a los participantes respecto a la cantidad de puntos ganados y de reportes correctos e incorrectos. En el monitor se presen- 
tó la siguiente información: "(nombre del sujeto): Ganaste XXX puntos de XXX puntos posibles, tuviste XX reportes correctos y XX reportes incorrectos".

Cuando los participantes lograron el criterio de la tarea pasaron a realizar las pruebas de relaciones condicionales emergentes (véase más adelante), en caso contrario se les programó otra sesión de discriminación.

A los participantes del grupo sin entrenamiento-con verbalización (SE-V) y del grupo con entrenamiento y con verbalización (E-V), que realizaron la tarea de discriminación condicional de la propia conducta verbalizando simultáneamente las contingencias, se les dieron verbalmente las siguientes instrucciones adicionales durante el componente de ejecución de cada ensayo:

"Dime en voz alta qué hay en la pantalla, qué hiciste y qué ocurrió cuando lo hiciste".

Los participantes de los grupos sin entrenamiento-sin verbalización (SE$\mathrm{SV}$ ) y con entrenamiento-sin verbalización (E-SV), realizaron la tarea de discriminación condicional de la propia conducta, sin instrucciones de verbalización simultánea de las contingencias.

Pruebas de relaciones condicionales emergentes de simetría, transitividad y equivalencia. En esta tercera condición experimental se aplicó, a todos los participantes, un conjunto de pruebas de discriminaciones condicionales emergentes o derivadas (véase la Figura 2), sin retroalimentación o reforzamiento de las ejecuciones bajo un formato de igualación de la muestra arbitraria simultánea.

Se evaluó la existencia de relaciones emergentes entre los tres componentes principales de la tarea de discriminación: rectángulo amarillo o azul, gráficas acumulativas de patrones en razón o en intervalo; y frases descriptivas de la ejecución más reciente (véase la Tabla 3 ).

En estas pruebas de relaciones condicionales emergentes, los participantes recibieron las siguientes instrucciones:

"En esta tarea, aparecerá un estímulo en la parte superior y luego otros dos estímulos en la parte inferior. (Se señaló la ubicación en el monitor de la computadora) Tienes que seleccionar uno de abajo que se relaciona con el de arriba, presionando la flecha izquierda o la derecha, según corresponda.

¿Tienes alguna duda?, dila al experimentador ahora."

En cada ensayo, aparecieron en la pantalla de la computadora tres estímulos (rectángulos de color azul o amarillo, frases descriptivas y gráficas acumulativas de las ejecuciones) en el formato habitual de igualación de la muestra. Los participantes tenían que presionar la tecla "flecha izquierda" o la tecla "flecha derecha", para seleccionar el estímulo de comparación que según ellos se relacionaba con el estímulo muestra. Luego la pantalla se aclaraba y medio segundo después aparecía la configuración de estímulos del siguiente ensayo. La posición de los estímulos de comparación se alternó aleatoriamente. 


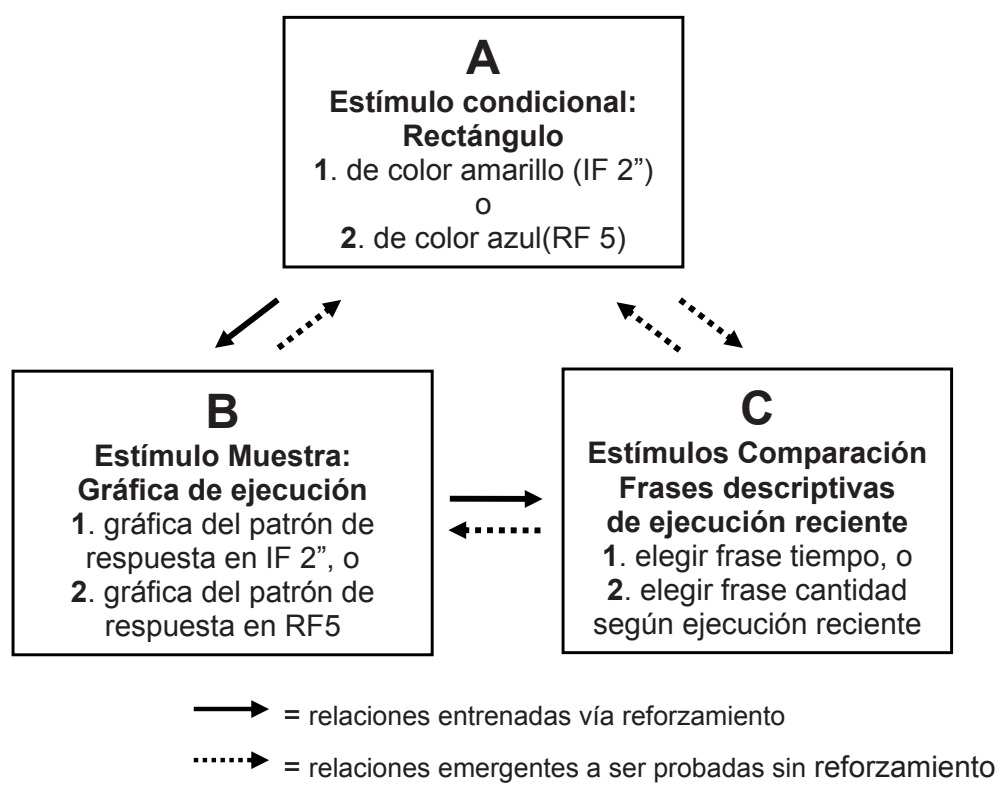

Nota: Las relaciones de línea base se establecieron con el formato de la estructura de la tarea de discriminación condicional de la propia conducta y las relaciones emergentes se probaron con un formato de igualación de la muestra arbitraria simultánea

Figura 2. Relaciones condicionales entrenadas y relaciones condicionales emergentes o derivadas entre los eventos estímulo de una discriminación condicional de la propia conducta.

En la Figura 3, se muestran ejemplos de los estímulos presentados en las pruebas de relaciones emergentes. Se tomaron los patrones de ejecución de los últimos ensayos de razón y de intervalo generados por los propios participantes, para usarlos como estímulos de muestra y de comparación en el procedimiento de igualación usado para las pruebas.

En virtud de que las pruebas se hicieron bajo el formato convencional de igualación de la muestra simultánea arbitraria, que es diferente del formato usado en este experimento para la adquisición de la discriminación condicional de la propia conducta, se realizaron doce pruebas de relaciones condicionales emergentes, que incluyeron a las relaciones condicionales de línea base (A1-B1, C2-B2, A2-C2, C1-A1, B1-A1, C1-B1, B2-A2, B2-C2, C2$A 2, A 1-C 1, B 1-C 1, A 2-B 2$; se adopta la nomenclatura convencional, donde el primer término de estos pares se refiere al estímulo muestra y el segundo al estímulo de comparación positivo). 


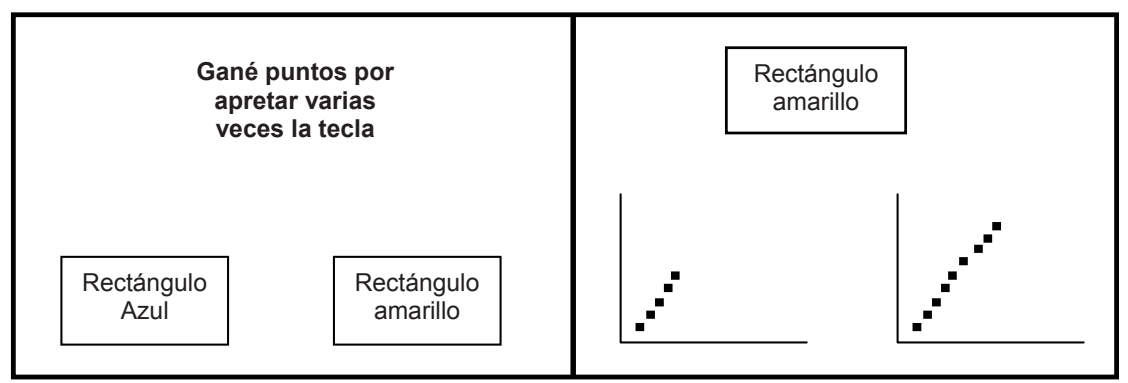

Figura 3. Ejemplos de configuración de estímulos presentados en las pruebas de relaciones condicionales emergentes, las pruebas se realizaron sin retroalimentación ni reforzamiento.

Tabla 3. Secuencia de las pruebas de relaciones condicionales emergentes y estímulos específicos presentados.

\begin{tabular}{|c|c|c|c|}
\hline $\begin{array}{c}\text { Secuencia de } \\
\text { pruebas y tipo de } \\
\text { prueba }\end{array}$ & $\begin{array}{l}\text { Estímulo } \\
\text { Muestra }\end{array}$ & $\begin{array}{l}\text { Comparación } \\
\text { Positivo }\end{array}$ & $\begin{array}{l}\text { Comparación } \\
\text { Negativo }\end{array}$ \\
\hline 1. A1-B1 & Rectángulo amarillo & $\begin{array}{l}\text { Gráfica patrón } \\
\text { intervalo }\end{array}$ & Gráfica patrón razón \\
\hline 2. $\mathrm{C} 2-\mathrm{B} 2$ & Descripción cantidad & Gráfica patrón razón & $\begin{array}{c}\text { Gráfica patrón } \\
\text { intervalo }\end{array}$ \\
\hline 3. A1-C1 & Rectángulo Azul & Descripción cantidad & Descripción tiempo \\
\hline 4. C2-A2 & Descripción tiempo & Rectángulo amarillo & Rectángulo azul \\
\hline 5. B1-A1 & $\begin{array}{c}\text { Gráfica patrón } \\
\text { intervalo }\end{array}$ & Rectángulo amarillo & Rectángulo azul \\
\hline 6. C1-B1 & Descripción tiempo & $\begin{array}{l}\text { Gráfica patrón } \\
\text { intervalo }\end{array}$ & Gráfica patrón razón \\
\hline 7. B2-A2 & Gráfica patrón razón & Rectángulo azul & Rectángulo amarillo \\
\hline 8. B2-C2 & Gráfica patrón razón & Descripción cantidad & Descripción tiempo \\
\hline 9. C1-A1 & Descripción cantidad & Rectángulo azul & Rectángulo amarillo \\
\hline 10. A2-C2 & Rectángulo amarillo & Descripción tiempo & Descripción cantidad \\
\hline 11. B1-C1 & $\begin{array}{c}\text { Gráfica patrón } \\
\text { intervalo }\end{array}$ & Descripción tiempo & Descripción cantidad \\
\hline 12. A2-B2 & Rectángulo Azul & Gráfica patrón razón & $\begin{array}{c}\text { Gráfica patrón } \\
\text { intervalo }\end{array}$ \\
\hline
\end{tabular}




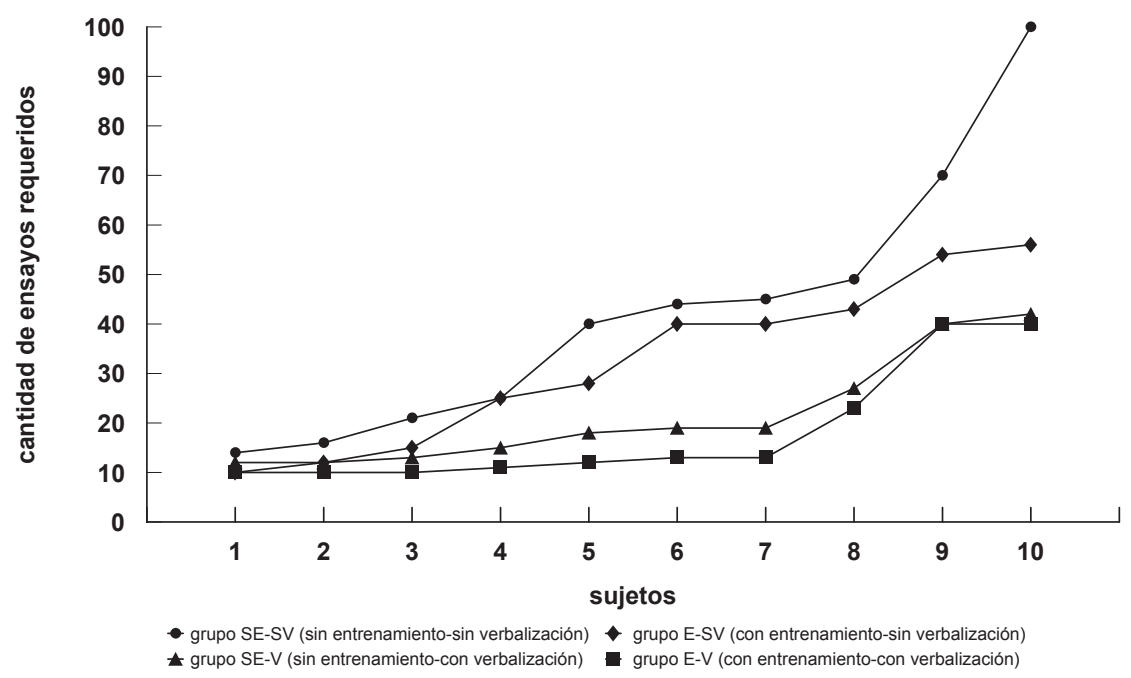

Figura 4. Comparación entre grupos experimentales de la cantidad total de ensayos requeridos por cada sujeto para la adquisición de la discriminación condicional de su propia conducta.

Cada prueba se integró con 10 ensayos. El criterio de formación de relaciones emergentes fue que los participantes tuvieran por lo menos 8 de 10 ensayos correctos en cada prueba. La secuencia de realización de las pruebas se determinó aleatoriamente.

Al término del experimento se explicó a los participantes el objetivo del mismo, las tareas que realizaron, sus resultados personales y se les agradeció su participación.

\section{Resultados}

Preentrenamiento. El 95\% de los sujetos sometidos a la condición de preentrenamiento en identificación y verbalización de relaciones contingentes logró el criterio de entrenamiento en una sola sesión, sólo uno de los sujetos requirió de dos sesiones para ello.

Adquisición de la discriminación condicional de la propia conducta. La Figura 4 compara por sujeto y grupo, la cantidad total de ensayos requeridos para la adquisición de una discriminación condicional de su propia conducta. Se observan diferencias claras entre todos los grupos experimentales.

Hay diferencias notables entre los grupos sin verbalización respecto de los grupos con verbalización. Los sujetos con verbalización requirieron de 18.2 
a 21.7 ensayos en promedio para adquirir la discriminación mientras que los sujetos sin verbalización necesitaron de 32.3 a 42.4 ensayos en promedio.

También se observan diferencias entre los grupos sin y con preentrenamiento. Hay dos claros agrupamientos determinados por la verbalización.

La adquisición de los grupos que no verbalizaron simultáneamente las contingencias (SE-SV y E-SV) fue más lenta, con rango de 10-100 ensayos; los grupos que verbalizaron simultáneamente las contingencias (SE-V y E-V) adquirieron la discriminación más rápidamente, con rango de 10-42 ensayos (recuérdese que el criterio de adquisición fue de 10 ensayos correctos continuos). El $100 \%$ de los sujetos de los grupos con verbalización adquirieron la discriminación condicional de su propia conducta entre 10-42 ensayos; mientras que el $60 \%$ de los sujetos de los grupos sin verbalización lograron el criterio en un rango de 10-42 ensayos, el otro $40 \%$ adquirió la discriminación en un rango de 43-100 ensayos.

Para corroborar las diferencias observadas, en términos de cantidad de ensayos requeridos para la adquisición de la discriminación, se realizó un Análisis de Varianza (ANOVA) con Entrenamiento y Verbalización como factores, donde sólo se encontraron efectos principales de Verbalización ( $\mathbf{F}$ $[1,36]=9.58, p=.004)$.

La Figura 5 compara la cantidad de ensayos correctos e incorrectos requeridos por cada sujeto para adquirir la discriminación condicional; se observan diferencias individuales pero con una clara consistencia intra-grupo. Los sujetos que verbalizaron las contingencias tuvieron entre 0-14 ensayos incorrectos, a diferencia de los sujetos de los grupos sin-verbalización que tuvieron entre $0-26$ ensayos incorrectos. El $80 \%$ de los errores de los grupos con verbalización estuvieron en el rango de 0-5 ensayos, mientras que tan solo el $40 \%$ de los errores de los grupos sin verbalización estuvo en este último rango.

Pruebas de relaciones condicionales emergentes. Se encontraron diferencias en el porcentaje de pruebas de relaciones condicionales emergentes aprobadas. Los grupos que no verbalizaron las contingencias tuvieron bajos porcentajes de aprobación; en tanto que, en uno de los grupos que sí verbalizaron las contingencias hubo un efecto de techo.

El grupo SE-SV aprobó el $57.5 \%$ de las pruebas, el grupo E-SV tuvo el $59.2 \%$ de pruebas aprobadas, el grupo SE-V tuvo el $85.8 \%$ y el grupo E-V tuvo el $100 \%$ de aprobación en las pruebas de relaciones condicionales emergentes. También hubo diferencias entre los grupos sin y con preentrenamiento, los primeros tuvieron porcentajes más bajos de pruebas aprobadas que los grupos con preentrenamiento, aquí las diferencias entre grupos son menores. 


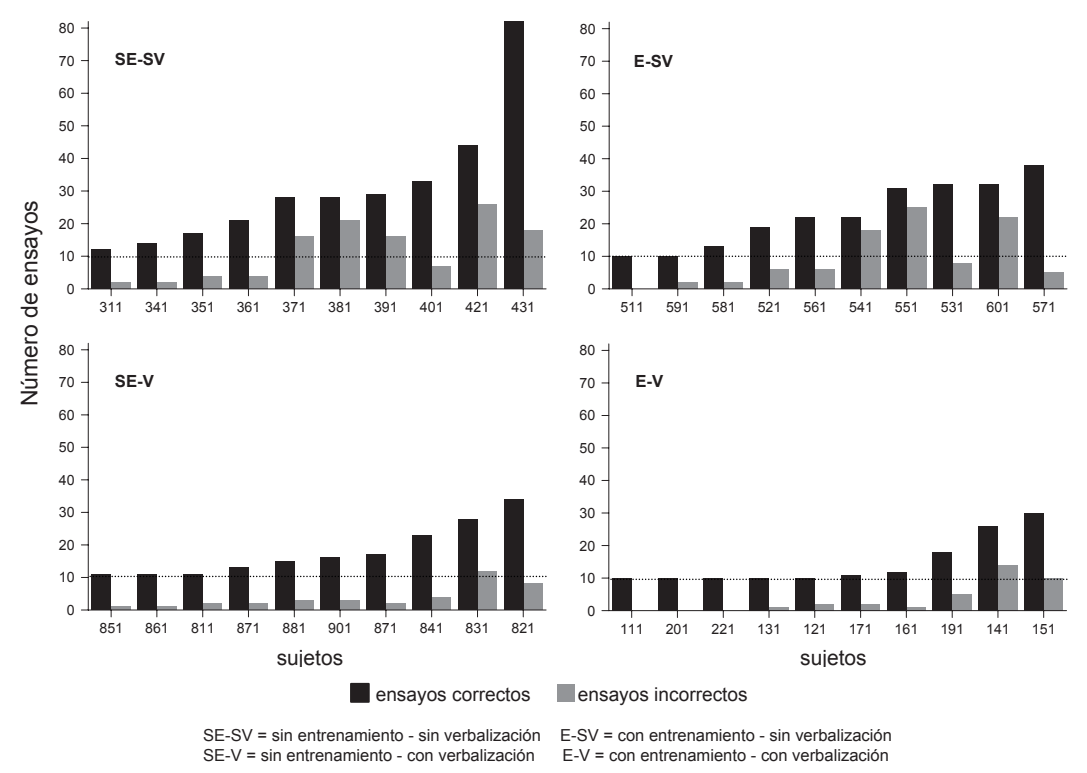

Figura 5. Cantidad de ensayos correctos e incorrectos requeridos por cada sujeto para la adquisición de la discriminación condicional de la propia conducta. En esta figura, los datos individuales se presentan de menor a mayor número de ensayos correctos para resaltar las diferencias. La línea punteada marca el criterio de adquisición.

La Figura 6 compara por sujeto y grupo, la cantidad de pruebas de relaciones condicionales emergentes aprobadas y no-aprobadas. Se observa que en los grupos sin-verbalización (SE-SV y E-SV), sólo 6 sujetos tuvieron entre $90-100 \%$ de pruebas aprobadas; mientras que en los grupos con-verbalización (SE-V y E-V), 18 sujetos tuvieron pruebas aprobadas en el rango de 90-100\%. También, se observa que en los grupos con entrenamiento (E-SV y E-V), 13 sujetos tuvieron entre el $90-100 \%$ de pruebas aprobadas; en tanto en los grupos sin entrenamiento (SE-SV y SE-V), 11 sujetos tuvieron entre 90-100\% de pruebas aprobadas. En este caso la diferencia es mínima.

Las Figuras 7 y 8 muestran el número de sujetos de cada grupo que aprobaron las relaciones condicionales emergentes por tipo de prueba. La Figura 7 compara los grupos sin y con verbalización. Las diferencias entre estos grupos es notable: se observa que un número mayor de sujetos (entre 17 y 19 sujetos) de los grupos con verbalización aprobó los distintos tipos de prueba; en tanto que un número menor de sujetos (entre 7 y 17 sujetos) de los grupos sin verbalización aprobó las distintas pruebas. 

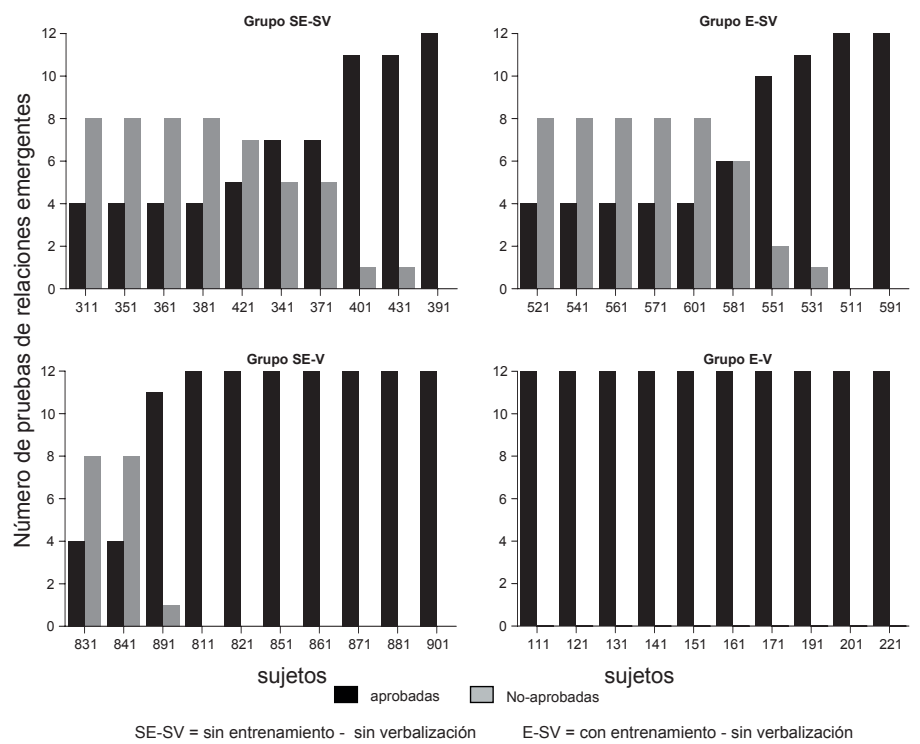
sujetos

$S E-S V=$ sin entrenamiento $-\sin$ verbalización
$S E-V=$ sin entrenamiento - con verbalización $\mathrm{E}-\mathrm{SV}=$ con entrenamiento - sin verbalización
$\mathrm{E}-\mathrm{V}=$ con entrenamiento - con verbalización

Figura 6. Comparación entre grupos experimentales de la cantidad de pruebas de relaciones emergentes aprobadas y no-aprobadas por cada sujeto.

La Figura 8 compara los grupos sin y con entrenamiento, en este caso las diferencias entre grupos son menores, sin embargo se observa que un número mayor de sujetos (entre 14 y 19 sujetos) de los grupos con entrenamiento aprobó en las relaciones condicionales emergentes; mientras que un número menor de sujetos (entre 11 y 16 sujetos) de los grupos sin entrenamiento aprobó las pruebas.

\section{Discusión}

Los resultados de la condición de preentrenamiento indican que, a pesar de que no se realizaron pruebas formales para evaluar sus capacidades, los sujetos participantes poseen capacidades auditivo-visuales y verbales que les permitieron identificar y verbalizar relaciones contingentes entre colores y tonos de distinta duración. Todos los sujetos excepto uno, lograron el preentrenamiento en una sola sesión.

Los resultados de la segunda condición experimental, la de adquisición de una discriminación condicional de la propia conducta, sugieren que la verbalización simultánea de las contingencias parece ser el factor determinante de las diferencias observadas. 


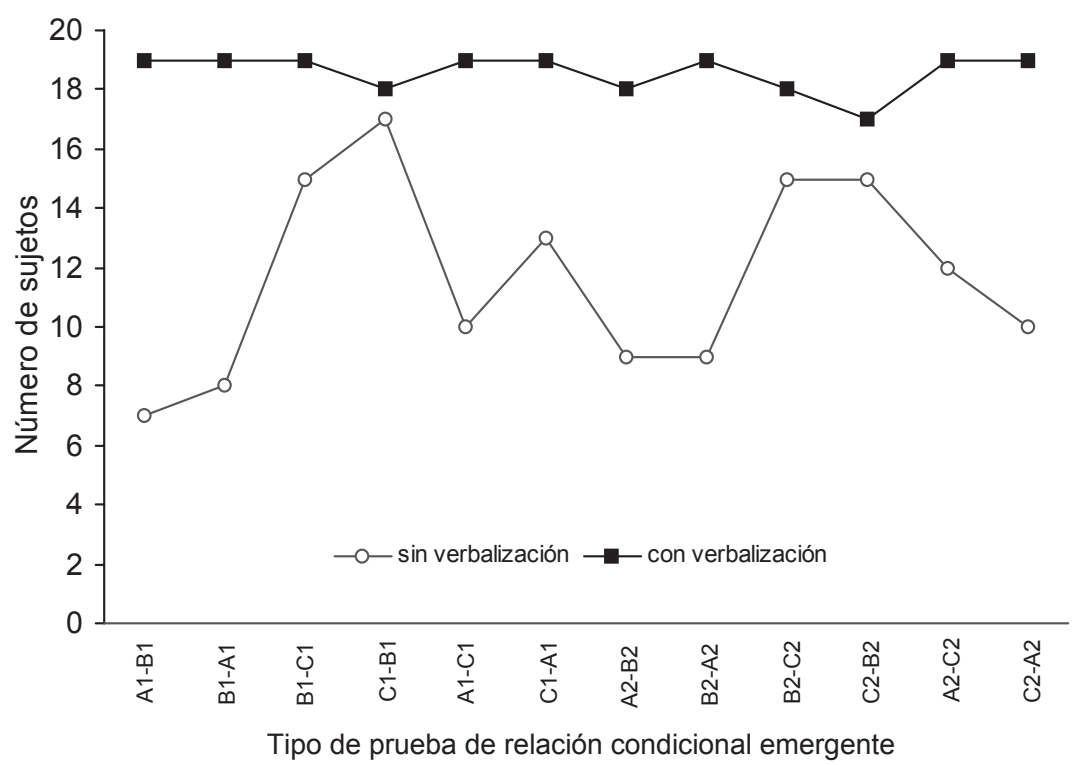

Figura 7. Comparación entre grupos sin y con verbalización del número de sujetos que aprobó las relaciones condicionales emergentes por tipo de prueba. El primer elemento de cada tipo de prueba representa al estímulo de muestra y el segundo elemento representa al estímulo de comparación positivo (véanse los detalles en la Tabla 3).

Recuérdese, que los sujetos simplemente tenían que decir en voz alta lo que veían en la pantalla, lo que hacían y lo que pasaba cuando hacían algo. Por ejemplo: "veo dos líneas, y un rectángulo de color azul. Presioné rápido y aparecieron varios cuadritos en lo que parece una gráfica".

Los sujetos que verbalizaron las contingencias adquirieron mucho más rápidamente la discriminación condicional de su propia conducta que los sujetos que no verbalizaron las contingencias, los segundos requirieron de un poco más del doble de ensayos para lograr la adquisición; esta diferencia fue corroborada por un ANOVA.

En cuanto al preentrenamiento, a pesar de que en la Figura 4, se observan diferencias visuales entre grupos sin y con preentrenamiento, el ANOVA no determinó efectos principales para este factor, ni para la interacción del preentrenamiento con la verbalización.

En la comparación del número de ensayos correctos e incorrectos requeridos para adquirir la discriminación de la propia conducta, se observó una alta consistencia intra-grupo. Es conveniente notar que una menor cantidad 


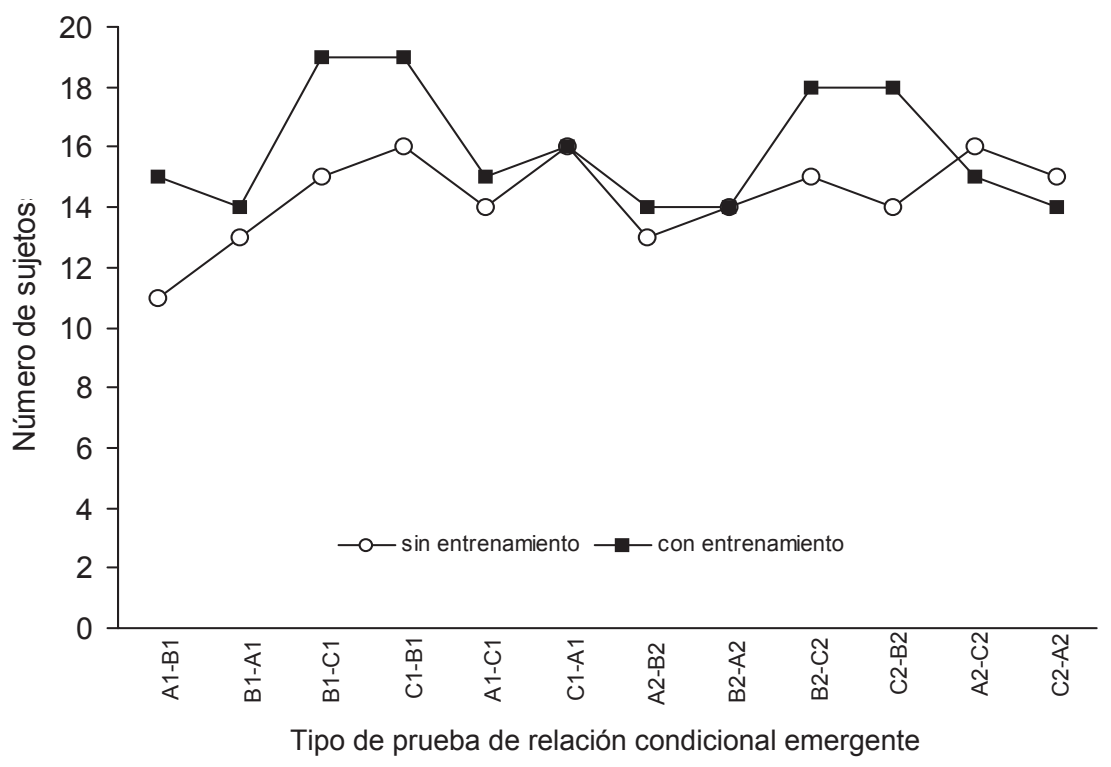

Figura 8. Comparación entre grupos sin y con entrenamiento del número de sujetos que aprobó las relaciones condicionales emergentes por tipo de prueba.

de ensayos correctos e incorrectos denota una mayor velocidad en la adquisición de la discriminación de la propia conducta. Los grupos con verbalización tuvieron un menor número de ensayos tanto correctos como incorrectos que los grupos sin verbalización, es decir adquirieron la discriminación más rápidamente.

En esta comparación, nuevamente se vuelve a observar que el factor verbalización parece determinar las diferencias; sin embargo, dos excepciones son dignas de discutir: el Sujeto 431 del Grupo SE-SV y el Sujeto 511 del Grupo E-SV.

El primero, fue el sujeto que requirió el mayor número de ensayos totales (100 ensayos y 4 sesiones) para adquirir la discriminación; el segundo, aparece fuera de la tendencia de su grupo ya que sólo necesitó el mínimo de 10 ensayos para adquirir la discriminación, es decir no tuvo ensayos incorrectos. Este hecho, sólo ocurrió en tres sujetos (111, 201 y 221) del grupo con entrenamiento y con verbalización, todos los demás sujetos tuvieron por lo menos un ensayo incorrecto.

El análisis de los errores del Sujeto 431, indica que todos los errores que cometió, excepto uno, fueron en componentes de elección que ocurrieron 
después de contingencias de intervalo, en las cuales el sujeto había dado por lo menos tres respuestas, por lo cual la gráfica acumulativa se asemejaba a la producida bajo contingencias de razón. Tal vez esa semejanza visual entre gráficas observadas le impidió al sujeto discriminar correctamente su ejecución y vincularla con la frase descriptiva pertinente. En su última sesión, el número de respuestas que dio en los ensayos de intervalo fue de entre 6 y 7 , lo cual configuraba una gráfica que era un poco más claramente discernible de la gráfica generada en los ensayos de razón.

Además, el análisis de las auto-descripciones que realizó el Sujeto 431 en los reportes post-sesión de sus primeras tres sesiones, indica que el sujeto relacionó "número de cuadritos con haber tecleado varias veces, si sólo aparecían 1 o 2 cuadros tomaba en cuenta el tiempo" (los "cuadritos" se refieren a los puntos que aparecían en la gráfica acumulativa cada vez que el sujeto presionaba la tecla flecha abajo). Estas descripciones muestran que el sujeto había identificado correctamente una parte de las contingencias (la relación entre propiedades del estímulo muestra y la elección de la frase descriptiva correcta correspondiente); sin embargo, en el reporte de su última sesión, en la que en tan sólo 10 ensayos adquirió finalmente la discriminación, describió las contingencias de la siguiente forma: "Cuando había un rectángulo azul es que presionaba varias veces la flecha de abajo. Y cuando había un rectángulo amarillo es que tardaba en presionar la flecha abajo".

El análisis del patrón de ejecución del Sujeto 511 , indica que en su primer ensayo, que fue de razón, su patrón de respuesta fue lento; y que el mismo patrón se mantuvo en sus ensayos siguientes. Este lento responder, durante los ensayos de intervalo, produjo un patrón de una sola respuesta; pues cuando el sujeto respondía ya había transcurrido el intervalo de 2 seg. Esto le permitió construir gráficas de su ejecución perfectamente diferenciadas: 5 respuestas muy espaciadas en razón, y 1 sola respuesta en intervalo.

La auto-descripción que realizó el Sujeto 511 en su reporte post-sesión, muestra que el sujeto describió las contingencias de la siguiente forma: "Tomé en cuenta las veces que había oprimido la tecla y el tiempo en que lo hice, después para elegir las respuestas escogí la que mejor describía lo que había hecho".

En lo general, los resultados de la segunda condición del presente experimento replican y extienden los hallazgos de Eikeseth y Smith (1992); Rehfeldt y Dixon (2000); Ribes y cols. (1996); Ribes y Castillo (1998); Ribes y cols. (1998) y Sato (2001). Los replican en el sentido de que los datos del presente experimento sugieren que la verbalización simultánea de las contingencias facilita una adquisición más rápida de la discriminación condicional de la propia conducta; los extienden en tanto que, en nuestro conocimiento, una tarea de discriminación condicional de la propia conducta como la propuesta, constituye una preparación experimental que previamente no se ha- 
bía empleado en el campo de estudio de los efectos del nombramiento sobre la adquisición de discriminaciones condicionales.

Como han señalado Martínez (1998), y López y Morales (1989), contar con dispositivos metodológicos a través de los cuales se obtenga la mayor cantidad de datos en el menor tiempo posible, es crucial en el campo de estudio de la conducta operante humana, en virtud de la imposibilidad de mantener a los sujetos humanos bajo situaciones controladas por períodos prolongados. Consideramos que con la tarea de discriminación condicional de la propia conducta se aporta un dispositivo metodológico que coadyuva a la solución de este problema.

Sin embargo, la conveniencia metodológica de la tarea de discriminación de la propia conducta propuesta, se debe tomar con reservas ya que no se comparó esta tarea con una situación en la cual todos los componentes de la discriminación sean dispuestos arbitrariamente por el experimentador.

Asimismo, los datos de nuestro experimento señalan que eventualmente todos los sujetos adquirieron la discriminación condicional de su propia conducta; esto indica que factores distintos a la verbalización abierta concurrente de las contingencias están operando en su adquisición.

Algunas posibilidades parecen viables: la adquisición se debe al mero efecto de las contingencias de reforzamiento dispuestas en la tarea; o bien la adquisición se debe al efecto, teóricamente posible, del nombramiento cubierto como han señalado Horne y Lowe (1996); o bien a un efecto conjunto del reforzamiento directo y de la verbalización abierta y/o cubierta.

Ribes y cols. (1998) han señalado que los sujetos, en su interacción con los procedimientos experimentales, pueden formular sus propias descripciones verbales que emergen paulatinamente en el transcurso del experimento. Esas auto-descripciones podrían influir tanto en la adquisición como en las pruebas de la emergencia de nuevas relaciones condicionales no entrenadas directamente.

Los resultados de la tercera condición experimental del presente experimento, la condición de prueba de relaciones condicionales emergentes indicativas de equivalencia, sugieren que la verbalización abierta y simultánea de las contingencias también tuvo efecto sobre la cantidad de pruebas de relaciones emergentes aprobadas. Todos los sujetos de los grupos con verbalización, excepto tres, aprobaron las doce pruebas de relaciones condicionales emergentes; a diferencia de los sujetos de los grupos que no verbalizaron las contingencias, en los que sólo tres sujetos aprobaron las doce pruebas.

A pesar de que las pruebas se aplicaron en un orden aleatorio, a diferencia de las secuencias empleadas comúnmente en las pruebas de simetría, de transitividad, y de equivalencia (así llamada por Sidman, 1992), los datos sugieren que en los repertorios de los sujetos que verbalizaron las contingencias y que adquirieron más rápidamente la discriminación condicional de su propia conducta, emergieron relaciones condicionales indicativas de equivalencia. 
Asimismo, los datos también sugieren que, las relaciones emergentes formaron dos clases estímulo de tres miembros cada una: la clase "tiempo o amarillo" integrada por los rectángulos de color amarillo, las gráficas de ejecución en intervalo y la frase "Gané puntos por el paso del tiempo y por apretar la tecla"; y la clase "cantidad o azul", integrada por el rectángulo azul, las gráficas de ejecución en razón y la frase "Gané puntos por apretar varias veces la tecla".

Es decir, las relaciones condicionales establecidas vía reforzamiento durante la adquisición de la discriminación de la propia conducta, parecen haber sentado las bases para la emergencia de nuevas relaciones condicionales no entrenadas directamente.

Para concluir, citamos una idea de Skinner (1969), que seguramente dio origen y guía al presente experimento: "Cualquier formulación actual de la relación entre una respuesta y sus consecuencias (quizás simplemente la observación 'siempre que respondo en esta forma, luego ocurren tales y tales eventos'), podría funcionar como un estímulo controlador previo" (p. 147).

\section{Referencias}

Catania, A. C. (1998). Learning. New Jersey: Prentice-Hall.

Constantine, B. y Sidman, M. (1975). Role of naming in delayed matching to sample. American Journal of Mental Deficiency, 79, 680-689.

Cumminng, W.W. y Berryman, R. (1965). The complex discriminated operant: Studies of matching-to-sample and related problems. En D.I. Mostofsky (Ed.), Stimulus generalization (pp. 284-330). Stanford, CA: Stanford University Press.

Dugdale, N. y Lowe, C. F. (1990). Naming and stimulus equivalence. En D. E. Blackman y $\mathrm{H}$. Lejeune (Eds.), Behavior analysis in theory and practice: Contributions and controversies (pp. 115-138). Hove, England: Erlbaum.

Eikeseth, S. y Smith, T. (1992). The development of functional and equivalence classes in high-functioning autistic children: The role of naming. Journal of the Experimental Analysis of Behavior, 58, 123-133.

Horne, P. J. y Lowe, C. F. (1996). On the origins of naming and other symbolic behavior. Journal of the Experimental Analysis of Behavior, 65, 185-241.

Keppel, G., Saufley, Jr., W. H. y Tokunaga, H. (1992). Introduction to design \& analysis. A student's handbook (2nd ed.). New York: Freeman.

Kerlinger, F. N. (1975). Investigación del comportamiento. Técnicas y metodología (2a Ed. en Español). México: Interamericana.

Lazar, R. M., Davis-Lang, D. y Sanchez, L. (1984) The formation of visual stimulus equivalence in children. Journal of the Experimental Analysis of Behavior, 41, 251-266.

López, F. Y Morales, F. (1989). Comportamiento humano en intervalo fijo: Un programa de retroalimentación con puntos. Revista Mexicana de Análisis de la Conducta, $15,73-85$.

Lowe, C. F. (1979). Determinants of human operant behavior. En M. D. Zeiler y P. 
Harzem (Eds.), Advances in analysis behaviour: Vol. 1. Reinforcement and the organization of behavior (pp. 159-192). Chichester, England: Wiley.

Lowe, C. F. (1983). Radical behaviorism and human psychology. En G. C. L. Davey (Ed.), Animal models of human behavior. Conceptual, evolutionary, and neurobiological perspectives (pp. 71-93). New York: John Wiley and Sons.

Martínez, H. (1998). Teoría de la conducta: Avances y perspectivas en la investigación del comportamiento humano. Acta Comportamentalia, 6, 99-111.

Randell, T. y Remington, B. (1999). Equivalence relations between visual stimuli: The functional role of naming. Journal of the Experimental Analysis of Behavior, 71, 395-415.

Rehfeldt, R. A. y Dixon, M. (2000). Investigating the relation between self-talk and emergent stimulus relations. Research in progress. Experimental Analysis of $\mathrm{Hu}-$ man Behavior Bulletin, 18, 28-29.

Ribes, E. y Castillo, A. (1998). Interacción del tipo de entrenamiento y el tipo de respuesta de igualación en transferencia en una discriminación condicional de segundo orden. Acta Comportamentalia, 6, 5-20.

Ribes, E., Moreno, D. y Martínez, C. (1998). Second-order discrimination in humans: The roles of explicit instructions and constructed verbal responding. Behavioural Processes, 42, 1-18.

Ribes, E., Torres, C. y Ramírez, L. (1996). Efecto de los modos de descripción en la adquisición y transferencia de una discriminación condicional de segundo orden en humanos adultos. Acta Comportamentalia, 4, 159-179.

Sato, T. (2001). The function of common naming and establishment of stimulus equivalence. Japanese Journal of Behavior Analysis, 16, 2-21.

Sidman, M. (1971). Reading and auditory-visual equivalences. Journal of Speech and Hearing Disorders, 14, 5-13.

Sidman, M. (1992). Equivalence relations: Some basic considerations. En S. C. Hayes y L. J. Hayes (Eds.), Understanding verbal relations. The second and third international institute on verbal relations ( pp. 15-27). Reno, Nevada: Context Press.

Sidman, M. y Cresson, O. (1973). Reading and crossmodal transfer of stimulus equivalence in severe retardation. American Journal of Mental Deficiency, 77, 515523.

Sidman, M., Cresson, O., Jr. y Willson-Morris, M. (1974). Acquisition to matching to sample via mediated transfer. Journal of the Experimental Analysis of Behavior, 22, 261-273.

Sidman, M., Rauzin, R., Lazar, R., Cunningham, S., Tailby, W. y Carrigan, P. (1982). A search of symmetry in the conditional discriminations of rhesus monkeys, baboons, and children. Journal of the Experimental Analysis of Behavior, 37, 23-44.

Sidman, M. y Tailby, W. (1982). Conditional discrimination vs. matching to sample: An expansion of testing paradigm. Journal of the Experimental Analysis of Behavior, $37,5-22$.

Sidman, M., Willson-Morris, M., y Kirk, B. (1986). Matching-to-sample procedures and development of equivalence relations: The role of naming. En M. Sidman (1994). Equivalence relations and behavior: A research story (pp. 281-320). Boston, MA: Authors Cooperative.

Skinner, B. F. (1969). Contingencies of reinforcement. New York: Appleton. 